\title{
High performance bidirectional electrostatic inchworm motor fabricated by trench isolation technology
}

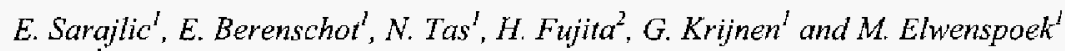 \\ ${ }^{1}$ MESA+ Research Institute, University Of Twente, The Netherlands \\ ${ }^{2}$ Institute of Industrial Science (IIS), University of Tokyo, Japan
}

\begin{abstract}
We report on an electrostatic linear micromotor, which employs built-in mechanical leverage to convert normal deflection of a flexible plate into a small in-plane step and two clamps to enable bidirectional inchworm motion. The motor, measuring $412 \mu \mathrm{m} \times 286 \mu \mathrm{m}$, is fabricated by a combination of trench isolation technology and standard surface micromachining in a relative simple process. The maximum achieved travel range was $\pm 70 \mu \mathrm{m}$, limited only by flexure design. Depending on the plate actuation voltage, two operation modes, below and above pull-in of the plate, are demonstrated with an adjustable step size from 0.6 to 7 am and 49 to $62 \mathrm{~nm}$, respectively. The motor was driven in a broad cycling frequency range from 0 to $80 \mathrm{kHz}$. Output forces of $1.7 \mathrm{mN}$ are measured at $55 \mathrm{~V}$ for both the clamps and plate. The motor was operated for 5 days at a stepping frequency of $80 \mathrm{kHz}$ and has completed a cumulative distance of more than $1500 \mathrm{~m}$ in about $34 \cdot 10^{9}$ steps without any performance deterioration.
\end{abstract}

Keywords: electrostatic microactuators, inchworm motion, shuffle motors, trench isolation

\section{INTRODUCTION}

Diverse MEMS applications e.g. probe-based data storage, microassembly, microscopy, robotics and optical systems require compact microactuators with relatively long displacement range, large output force, high speed and fine positioning resolution.

Shuffle motors $[1,2]$ are potential candidates to satisfy these diverse requirements. Shuffle motors employ built-in mechanical leverage to convert normal deflection of an elastic plate into a small powerful step and two electrostatic clamps to enable bidirectional inchworm motion.

The first shuffle motor was fabricated by surface micromachining [1]. The motor, measuring $560 \mu \mathrm{m} \mathrm{x} 400$ $\mu \mathrm{m}$, was driven successfully at maximum speed of 0.1 $\mathrm{mm} / \mathrm{s}$, corresponding with a cycling frequency of $1.16 \mathrm{kHz}$ and an average step size of $85 \mathrm{~nm}$. An output force of 0.043 $\mathrm{mN}$ was achieved at the plate voltage of $25 \mathrm{~V}$ and clamp voltage of $40 \mathrm{~V}$. Performance and reliability of the motor was limited due to the charge accumulation in the insulating silicon nitride layer.

The negative effect of charge accumulation was eliminated in an improved shuffle motor design by only allowing mechanical contact between electrically grounded parts [2]. The motor, measuring $200 \mu \mathrm{m} \times 1500 \mu \mathrm{m}$, has achieved a force of $0.45 \mathrm{mN}$ at driving voltages of $65 \mathrm{~V}$ and $150 \mathrm{~V}$ for the plate and clamps, respectively. A large stroke of \pm 70 $\mu \mathrm{m}$, large velocity range up to $4.4 \mathrm{~mm} / \mathrm{s}$ and an average step size of $100 \mathrm{~nm}$ are demonstrated. The motor was fabricated using a five-layer polycrystalline silicon surface micromachining (SUMMiT V technology).

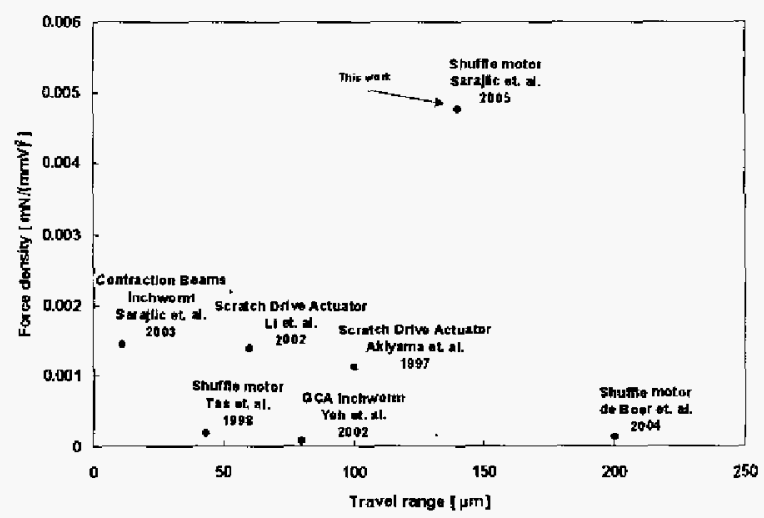

Figure 1: Force density normalized to the driving voltage squared versus travel range of several large force electrostatic microactuators $[1-6]$.

In this paper we realize a shuffle motor using a combination of trench isolation technology and standard surface micromachining. This enables us to simplify the fabrication process and improve overall performance characteristics and reliability. The presented shuffle motor, we believe, has the highest force density $\left(\mathrm{mN} / \mathrm{mm}^{2} / \mathrm{N}^{2}\right)$ ever published for an electrostatic microactuator, as shown in Fig. 1.

\section{DESIGN}

Fig. 2 shows the design of the shuffle motor.

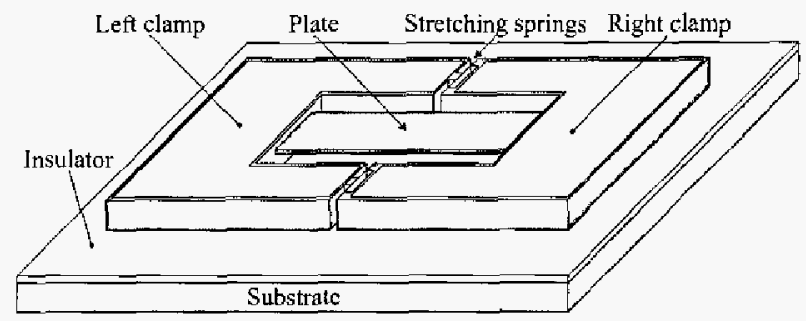

Figure 2: A schematic view of a shuffle motor.

The motor consists of two $\mathrm{U}$-shaped clamps and an elastic plate. The stretching springs between the clamps are

\section{TRANSDUCERS'05}

The 13th International Conference on Solid-State Sensors, Actuators and Microsystems, Seoul, Korea, June 5-9, 2005 
employed to guide the relative motion of the clamps. The plate and clamps are mutually electrically insulated, allowing individual biasing of each of these components. The motor moves over an unpattemed substrate, covered by an insulating layer. The substrate is electrically grounded during operation of the motor. Driving voltage signals to the clamps and plate are provided through the suspension of the motor, which is not shown in Fig. 2.

The proposed design is substantially different from the previously reported shuffle motors $[1,2]$, where the whole motor is grounded and driving voltage signals are provided through underlying electrodes. In our design, motion of the motor is not restricted to predefined paths, allowing large freedom of motion. In addition, the fabrication steps required for the underlying electrodes can be omitted, reducing the complexity of the fabrication process. Finally, omitting underlying electrodes gives the possibility to realize a shuffle motor with two degrees of freedom [7].

By applying an appropriate voltage sequence a walking motion of the shuffle motor is achieved, as illustrated in Fig. 3.

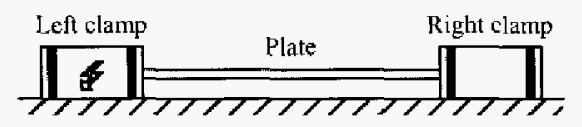

(I)

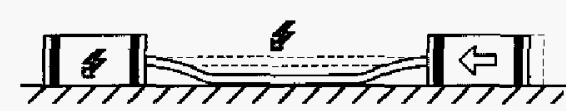

(II)

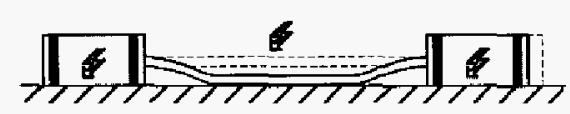

(III)

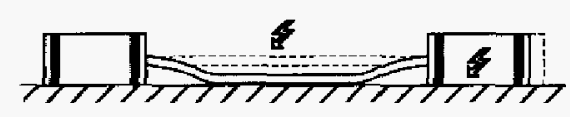

(IV)

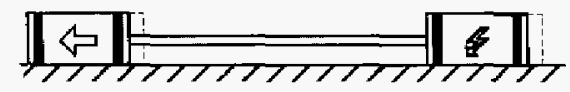

(V)

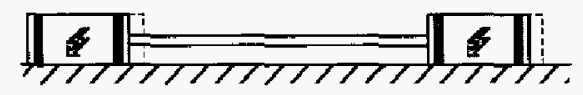

(VI)

Figure 3: Voltage actuation sequence of a shuffle motor.

First, a voltage is applied on the left clamp, inducing an electrostatic force that prevents sliding of the activated clamp (I). Next, a voltage difference is applied between the plate and the substrate, causing deflection of the plate. The normal deflection of the plate induces a contraction of the entire motor, moving the right clamp closer to the left $(I I)$. Since the normal deflection of the plate is much larger than the induced longitudinal motion there is a built-in mechanical leverage, resulting in a small but powerful contraction. Subsequently, the right clamp is activated in the new position (III) and the left clamp is then released (IV). The released plate stretches and pushes the left clamp outwards $(V)$. The actuation sequence is completed by activation of the left clamp (VI). The whole motor is moved a single step to the left. By repeating the actuation sequence a large number of steps can successively be added, resulting in a large displacement range. By reversing the sequence inchworm motion in the opposite direction can be achieved.

\section{FABRICATION}

A successful realization of the proposed shuffle motor requires a proper electrical insulation and, at the same time, mechanical connection between the clamps and the plate. We combine vertical trench isolation with conventional surface micromachining to satisfy these requirements within a minimum number of processing steps.

A relative simple fabrication process, which employs only two polycrystalline silicon device layers and four photolithography masks, is developed. Fig. 4 illustrates the cross-section of the shuffle motor after release. A detailed description of all processing steps is given in Ref. [7].

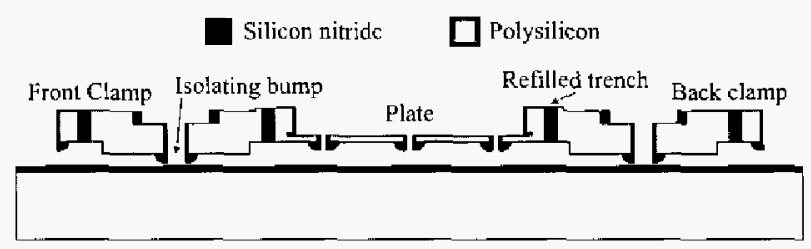

Figure 4: Schematic view of the cross section of the shuffle motor after release step.

The motor employs trenches refilled with silicon nitride to assure electrical insulation between the clamps and plate. Isolating trenches enable independent biasing of these components. At the same time, a high stiffness and large mechanical strength of isolating trenches assure mechanical integrity of the motor. Silicon nitride isolating bumps are evenly spaced on the backsides of the clamps and plate. The isolating bumps reduce the contact area significantly, preventing stiction of the clamps and plate during fabrication and operation. During operation of the shuffle motor only isolating bumps make contact with the silicon nitride coating on the wafer surface, reducing contact charging and charge accumulation. Furthermore, silicon nitride/silicon nitride contact (two hard materials) reduces wear, increasing reliability of the motor.

A successfully fabricated shuffle motor, with all components, is shown in Fig. 5. The entite motor, including two clamps and the plate, fits in an area of $482 \mu \mathrm{m} \times 286$ $\mu \mathrm{m}$. The motor is suspended by connection springs, which guide its motion and provide voltage signals to the clamps and plate. The springs are realized in a $5.5 \mu \mathrm{m}$ thick polysilicon layer and suspended $1.95 \mu \mathrm{m}$ above the coated surface. The connection springs are not electrically shielded from the grounded substrate, therefore their dimensions

TRANSDUCERS'05

The 13th International Conference on Solid-State Sensors, Actuators and Microsystems, Seoul, Korea, June 5-9, 2005 
must be carefully chosen to prevent pull-down to the substrate during operation.

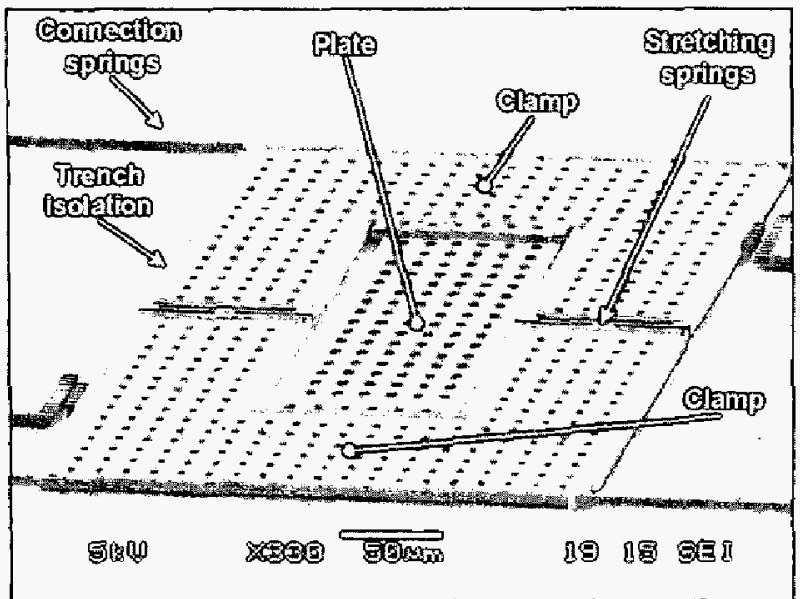

Figure 5: SEM micrograph of a shuffle motor with all components.

Dimensions of the shuffle motor are given in Table 1.

Table 1: Dimensions of the shuffle motor.

\begin{tabular}{lc}
\hline \multicolumn{1}{c}{ Parameter } & Dimensions \\
\hline \hline Area of motot & $412 \mu \mathrm{m} \times 286 \mu \mathrm{m}$ \\
Plate length & $180 \mu \mathrm{m}$ \\
Plate width & $94 \mu \mathrm{m}$ \\
Plate thickness & $1.15 \mu \mathrm{m}$ \\
Gap plate/substrate & $1.76 \mu \mathrm{m}$ \\
Bump height & $210 \mathrm{~nm}$ \\
Insulating layer thickness & $210 \mathrm{~nm}$ \\
Area of a clamp & $38.10^{3} \mu \mathrm{m}^{2}$ \\
\hline
\end{tabular}

\section{MEASUREMENTS}

We have successfully generated inchworm motion with the fabricated shuffle motor using a coordinated sequencing of the clamps and plate. An appropriate voltage scquence is generated using a multichannel analog output card and high voltage amplifier with high slew rate. The maximum displacement range of the motor was limited to $\pm 70 \mu \mathrm{m}$ by suspension design.

The positioning performance of the motor was measured using an image processing technique, based on the Fourier transform. This technique allows in-plane sub-pixel displacement measurements with a resolution of a few nanometers. Fig. 6 shows a typical measurement of the motor position versus time. The motor was actuated at $1 \mathrm{~Hz}$ stepping frequency using a driving voltage of $45 \mathrm{~V}$ for both the clamps and plate. Duc to the high resolution of the measurement, the separate steps can be clearly distinguished.

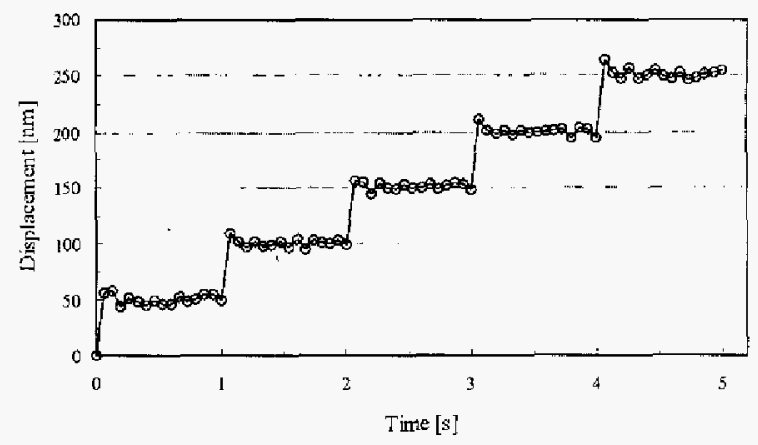

Figure 6: Displacement of a shuffe motor versus time. Data were taken at a stepping frequency of $1 \mathrm{~Hz}$ and a voltage of $45 \mathrm{~V}$ for both the clamps and plate.

Speed measurements were performed by operating the motor at a certain frequency for a fixed number of steps (100 steps) and measuring the distance traveled [2]. The speed is then calculated by dividing the traveled distance by the time of operation, derived from the cycling frequency and the number of steps. For each frequency, we took the average value of three measurements. The average deviation for each measurement point was smaller than $0.1 \%$. Fig. 7 shows the speed of the shuffle motor as a function of the stepping frequency. The data were taken at frequencies up to $80 \mathrm{kHz}$ with a motor actuated at $45 \mathrm{~V}$ for both the clamps and plate. The measured frequency range of $80 \mathrm{kHz}$ was limited by the available driving electronics.

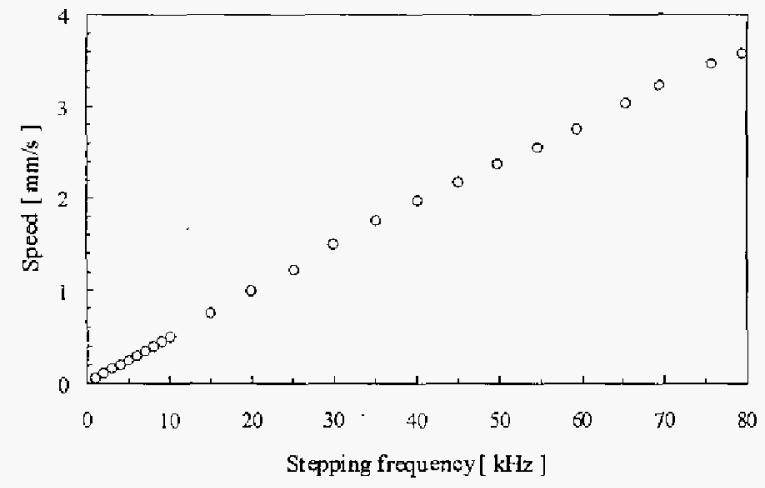

Figure 7: Measured speed of the shuffle motor versus stepping frequency. Measurement was performed at clamp and plate potentials of $45 \mathrm{~V}$.

The velocity is fairly linear with cycling frequency over the measured range with a maximum velocity of $3.6 \mathrm{~mm} / \mathrm{s}$ at $80 \mathrm{kHz}$. The slope of the speed curve is a measure for the average step size, which was about $50 \mathrm{~nm}$.

The velocity of the shuffle motor depends not only on the cycling frequency but also on the average step size. The step size of the motor is a function of the degree of plate

\section{TRANSDUCERS'05}

The 13th International Conference on Solid-State Sensors, Actuators and Microsystems, Seoul, Korea, June 5-9, 2005 
deformation, which is directly related to the plate actuation voltage, as shown in Fig. 8.

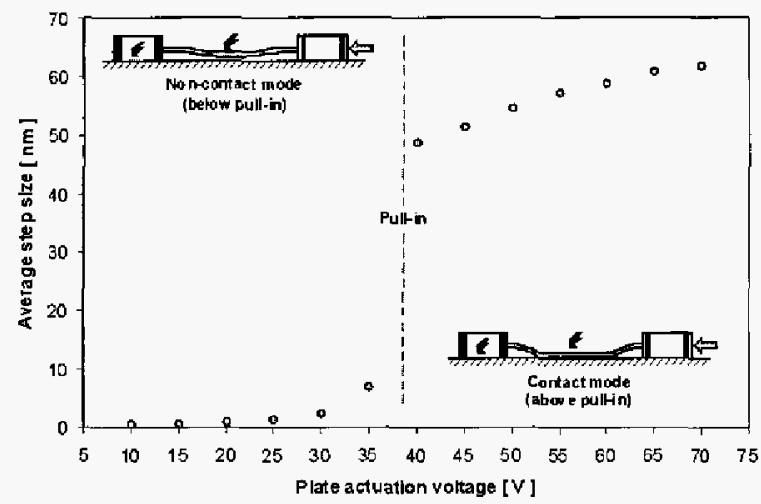

Figure 8: Measured average step size versus plate actuation voltage. Clamp voltage of $30 \mathrm{~V}$ and cycle frequency of 200 Hz are used.

We have shown an average step size from 0.6 to $7.0 \mathrm{~nm}$ for the motor operated below the pull-in voltage of the plate of $38 \mathrm{~V}$. By operating the plate above pull-in instability, collapse of the plate on the substrate occurs, resulting in a large plate deformation and significant increase of the step size. We have measured adjustable nanometer-resolution steps ranging from $49 \mathrm{~nm}$ to $62 \mathrm{~nm}$, for plate voltages ranging between $40 \mathrm{~V}$ and $70 \mathrm{~V}$. The measurements are performed at cycling frequency of $200 \mathrm{~Hz}$ and a clamping voltage of $30 \mathrm{~V}$.

Using in-plane displacement measurements and calculated stiffness of the suspension, we have determined the output force of the motor. Since the motor works against half of the connection springs at the same time (an activated clamp balance the spring force of the connection springs attached on it), the half stiffness of the suspension was used in the calculation.

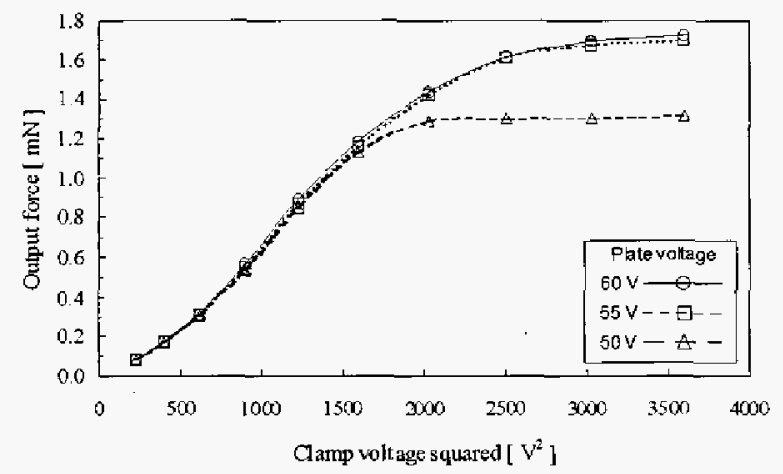

Figure 9: Measured output force of the shuffle motor versus clamp voltage for different plate actuation voltages at cycle frequency of $200 \mathrm{~Hz}$.
Fig. 9 shows the output force as a function of the clamp voltage squared for plate voltages of $50 \mathrm{~V}, 55 \mathrm{~V}$ and $60 \mathrm{~V}$. A maximum output force of $1.7 \mathrm{mN}$ is measured using actuation voltages of $55 \mathrm{~V}$ on both clamps and plate. By increasing the plate voltage further to $60 \mathrm{~V}$, the maximum output force did not alter.

A lifetime test was conducted to determine the durability of the shuffle motor. The motor was run for five days at a cycling frequency of $80 \mathrm{kHz}$, generating nearly 34 billion steps, which is equivalent to a displacement of $1500 \mathrm{~m}$, without any noticeable change in performance.

\section{CONCLUSION}

A bidirectional electrostatic linear inchworm micromotor is presented. Small size and high performance characteristics in terms of output force, positioning resolution, speed and durability makes this motor a promising candidate for diverse MEMS applications.

\section{ACKWNOLEGMENTS}

The authors would like to thank M. de Boer, R. Sanders, T. Lammerink and $\mathrm{MESA}^{+}$clean room staff for their contribution to the fabrication and measurements. A part of the research, presented in this paper, was performed at Fujita Lab, University of Tokyo, Japan. All lab members are gratefully acknowledged. This work is a part of the research program on Micro. Scanning Probe Array Memory ( $\mu$ SPAM) supported by the Dutch Technology Foundation (STW).

\section{REFERENCES}

[1] Tas N, Wissink J, Sander L, Lammerink $T$ and Elwenspoek M 1998 Modeling, design and testing of the electrostatic shuffle motor Sensors and Actuators $A 70171$ 178

[2] de Boer M P, Luck D L, Ashurst W R, Maboudian R, Corwin A D, Walraven J A, Redmond J M 2004 High performance surface-micromachined inchworm actuator $J$. Microelectormech. Syst. 13 63-74

[3] Akiyama T, Collard D and Fujita H 1997 Scratch drive actuator with mechanical links for self-assembly of threedimensional MEMS J. Microelectormech. Syst. 6(1) 10-17 [4] Li L, Brown G and Uttamchandani 2002 Study of scratch drive actuator force characteristics J. Micromech. Microeng. $12736-741$

[5] Yeh R, Hollar S and Pister K S J 2002 Single mask, large force and large displacement electrostatic linear inchworm motors $J$. Microelectormech. Syst. 11(4) 330-336 [6] Sarajlic E, Berenschot E, Krijnen $G$ and Elwenspoek 2003 A low volume electrostatic inchworm microactuator with high-resolution and large force 17th European Conference on Solid-State Transducers (EUROSENSORS) (Guimaraes, Portugal) 194-195

[7] Sarajlic E, Berenschot E, Fujita H, Krijnen G and Elwerspoek 2003 Bidirectional electrostatic linear shuffle motor with two degrees of freedom 18th Int. Conf. On MEMS (MEMS 2005) (Miami, USA) 391-394 